\title{
Innovations en approches endoscopiques mini-invasives diagnostique et interventionnelle Conclusion
}

\author{
E. Coron · G. Meurette \\ (C) Lavoisier SAS 2014
}

Endoscopie et chirurgie mini-invasive se rejoignent progressivement dans le domaine de la colo-proctologie. L'endoscopie est un outil incontournable pour faire progresser le diagnostic des affections colorectales mais également (désormais) leur prise en charge. Ces avancées récentes font progresser la prise en charge des patients et surtout évitent de plus en plus des chirurgies délabrantes. L'exemple de l'intervention de Malone progressivement remplacée par la caecostomie endoscopique traduit cette évolution. D'autres domaines de la colo-proctologie ne sont pas traités dans ce dossier mais la liste est longue... La résection endoscopique transanale pour les tumeurs du rectum est un autre exemple prometteur. La formation des chirurgiens et des endoscopistes à ces dispositifs innovants est cruciale. Simulation est un terme auquel nous devrons nous habituer et intégrer à nos pratiques avant d'entreprendre des procédures nouvelles et innovantes.

Ce dossier thématique " nouveautés en coloproctologie interventionnelle et diagnostique » aborde ces différents thèmes. Nous espérons qu'il aura effectivement permis d'éveiller la curiosité et la réflexion sur notre avenir à tous quel que soit notre domaine d'exercice... 